\title{
QUANTIFICATION OF SEGREGATION IN PORTLAND CEMENT CON- CRETE BASED ON SPATIAL DISTRIBUTION OF AGGREGATE SIZE FRACTIONS
}

\author{
MURAT OZEN ${ }^{\bowtie, 1}$, MURAT GULER $^{2}$ \\ ${ }^{1}$ Mersin University, Department of Civil Engineering, Mersin, Turkey; ${ }^{2}$ Middle East Technical University, De- \\ partment of Civil Engineering, Ankara, Turkey \\ e-mail: ozen.murat@mersin.edu.tr, gmurat@metu.edu.tr \\ (Received December 27, 2019; revised July 16, 2020; accepted July 23, 2020)
}

\begin{abstract}
Segregation is one of the quality standards that must be monitored during the fabrication and placement of Portland cement concrete. Segregation refers to separation of coarse aggregate from the cement paste, resulting in inhomogeneous mixture. This study introduces a digital imaging based technique to quantify the segregation of Portland cement concrete from 2D digital images of cut sections. In the previous studies, segregation was evaluated based on the existence of coarse aggregate fraction at different geometrical regions of a sample cross section without considering its distribution characteristics. However, it is shown that almost all particle fractions can form clusters and increase the degree of segregation, thus deteriorating the structural performance of concrete. In the proposed methodology, a segregation index is developed by based on the spatial distribution of different size fractions of coarse aggregate within a sample cross section. It is shown that degradation in mixture's homogeneity is controlled by the combined effect of particle distribution and their relative proportions in the mixture. Hence, a segregation index characterizing the mixture inhomogeneity is developed by considering not only spatial distribution of aggregate particles, but also their size fractions in the mixture. The proposed methodology can be successfully used as a quality control tool for monitoring the segregation level in hardened concrete samples.
\end{abstract}

Keywords: Concrete, digital imaging, segregation, uniformity.

\section{INTRODUCTION}

The aggregate phase occupies up to $80 \%$ of concrete volume, leading to significant influence on both fresh and hardened properties of concrete. The size distribution of aggregate particles is one of the key parameters of concrete design affecting the strength and workability properties of the mixtures. Apart from the initial design, maintaining the design size distribution during manufacturing and placement is critical to achieve desired structural performance of concrete. Segregation is one of the quality standards that must be monitored during the placement of concrete. It refers to separation of coarse aggregate from the cement paste, resulting in an inhomogeneous mixture. Segregation can occur due to either settling or separation of coarse aggregate fraction in the mixture as a result of improper placing or vibration during the manufacturing process (Mindess et al., 2002). A highly segregated mixture will display decreased homogeneity leading to greater variability in the strength properties because of inadequate internal structure $(\mathrm{Na}-$ varrete and Lopez, 2016). Because the reduced strength and durability are the consequences of segregation in
Portland cement concrete, satisfying the quality requirements of concrete by controlling segregation becomes critical for achieving adequate mechanical properties, hence higher structural performance (Ferraris et al., 2008; Mesbah et al., 2011). Although the conventional methods for measuring segregation consider only fresh properties of concrete, they don't use standard techniques for monitoring the segregation properties in hardened concrete. Evaluation of segregation in the hardened phase is also important to confirm the test results for fresh concrete and can be used as a quality control tool when the test for fresh phase segregation is not performed (Navarrete and Lopez, 2016).

Recently, digital imaging methods has found a great interest in studying micro-structural characteristics, particle size distribution, size and shape characteristics and air void distribution of concrete (Yang et al., 2014; Nichols and Lange, 2006; Mora and Kwan, 2000; Ozen and Guler, 2014; Fernlund et al., 2007; Lee et al., 2007; He et al., 2016; Peterson et al., 2001). A main advantage of image processing relies on its ability to perform such analysis in a rapid, objective, efficient and cost effective 
manner with moderate level of hardware and software requirements. However, studies focusing on the analysis of segregation in hardened concrete are still limited and offer impractical methodologies. In a recent study by Solak et al. (2018), a segregation index was developed for lightweight concrete from the digital images of horizontal cut sections. Barbosa et al. (2011) proposed an index to evaluate distribution of aggregate particles in lightweight concrete. Fang and Labi (2007) developed an automated imaging methodology to identify the location of aggregate particles and mortar layer thickness for the study of segregation in self-consolidating concrete (SCC). In another study by Khayat et al. (2007), cylindrical samples of SCC were cut vertically and each section was further horizontally divided into six sections and then the percentage of coarse aggregate areas from top to bottom sections were calculated to evaluate the segregation. Johnson et al. (2010) calculated the ratio of cement area between top and bottom sections from the digital images of horizontal cut sections to quantify segregation in SCC samples. Erdem (2014) used digital images obtained from X-ray computed tomography to study segregation in SCC concrete samples by computing the volume of coarse aggregate particles in the upper and lower parts of the vertical and horizontal cut sections.

In these previous studies, segregation phenomena were considered by evaluating the percentage of coarse aggregate particles and cement paste at different sections of concrete samples. However, the main problem in these methods is the lack of considering the spatial distribution of coarse aggregate fractions within the cross sections. Even if the coarse aggregates are homogeneously distributed in the cement paste, larger or smaller coarse particles can form clusters within the sample cross section, thus affecting the structural performance of concrete. In this study, a digital imaging based technique is introduced to quantify the segregation of Portland cement concrete from 2D digital images of cut sections. In the proposed methodology, a segregation index is developed by considering the spatial distribution of different size fractions of coarse aggregate within a sample cross section. It is believed that the proposed methodology can be successfully used as a quality control tool for monitoring the segregation level in hardened concrete samples.

\section{MATERIAL AND METHODS}

\section{MIXTURE PROPORTIONS}

This study was conducted using two different concrete mix designs fabricated with natural crushed aggregates of $D_{\max }=13 \mathrm{~cm}$ and $25 \mathrm{~cm}$. The selected gradations can be found in Table 1. Standard $150 \mathrm{~mm}$ cubical concrete samples with five replicates were prepared for each mix design combination. The purpose of using different maximum aggregate sizes was to evaluate the sensitivity of the proposed methodology.

Table 1. Particle size distribution of aggregates used in the mix designs

\begin{tabular}{|c|c|c|}
\hline \multirow{2}{*}{$\begin{array}{l}\text { Sieve } \\
\text { size } \\
(\mathrm{mm})\end{array}$} & \multicolumn{2}{|c|}{$\begin{array}{c}\text { Percent passing } \\
\text { by weight }\end{array}$} \\
\hline & $\begin{array}{l}\text { Mix De- } \\
\text { sign } 1 \\
(\%)\end{array}$ & $\begin{array}{c}\text { Mix De- } \\
\text { sign } 2 \\
(\%)\end{array}$ \\
\hline 25.4 & 100.0 & 100.0 \\
\hline 19.1 & 100.0 & 85.0 \\
\hline 12.7 & 100.0 & 72.0 \\
\hline 9.5 & 87.0 & 60.0 \\
\hline 4.76 & 60.0 & 45.0 \\
\hline 2.38 & 44.8 & 33.0 \\
\hline 1.19 & 31.5 & 23.0 \\
\hline 0.59 & 15.4 & 11.0 \\
\hline 0.297 & 14.2 & 10.1 \\
\hline 0.149 & 13.5 & 9.8 \\
\hline
\end{tabular}

\section{IMAGE ACQUISITION AND OPTIMUM THRESHOLD}

After the curing period, each cubical sample was cut into four equal pieces using a circular diamond saw. For each sample, this process produced a total of six cross sectional surfaces with three overlapping cut sections as presented in Fig. 1. However, only one of the overlapped cut surfaces was used to avoid duplicating the image analysis of the same cross section. After the cutting process, the cross sections were digitized using a desktop flatbed scanner at a resolution of $150 \mathrm{dpi}$ in order to keep the file size moderate for further processing and analysis. Therefore, the pixels' size in digitized images were $0.169 \mathrm{~mm} \times 0.169 \mathrm{~mm}$. The digital images were stored in gray scale format in which every pixel has a color depth ranging from 0 to 255 , i.e., "0" indicates black and " 255 " as white.

Analysis of cross sectional images starts with the implementation of gray scale segmentation. Thresholding is one of the commonly used image segmentation methods in which objects of interest are extracted from the background by selecting a threshold value $(\mathrm{T})$, which varies between 0 and 255 for a gray scale image. Any pixel value lower than the threshold is classified as object pixel; otherwise, it belongs to the background of the image frame. In this study, an optimum threshold method previously developed by the authors was applied 
to successfully detect aggregate particles within a cross sectional image (Ozen and Guler, 2014).

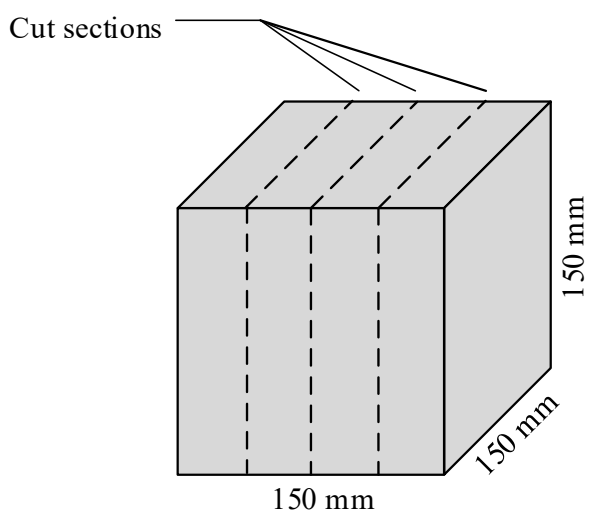

Fig. 1. Concrete sample cut sections

The resultant binary images may need morphological processing due to the existence of noise speckles, particles touching the border of image frames and overlapped particles. The number of erosion-dilation (opening) cycles with a $3 \times 3$ matrix of ones was applied to remove these artifacts and separate overlapped particles in the binary images. The removal of the small particles does have any negative impact on the results, as the focus of the study is the particles larger than $4.76 \mathrm{~mm}$ that cannot be removed during the erosion-dilation cycles.

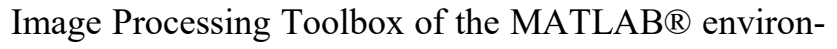
ment was used to calculate the optimum threshold for segmentation and perform and the erosion-dilation operations. Fig. 2 illustrates the original cross sectional image on the left, and its binary phase on the right after the implementation of the erosion-dilation cycles.

\section{PARTICLE SIZE DISTRIBUTION ANALYSIS}

To evaluate the segregation of concrete samples from $2 \mathrm{D}$ cross sectional images, sieve sizes to be used for calculating the size fraction of aggregate particles need to be determined. Traditionally, the gradation of aggregate samples is determined by mechanically sieving the material through a series of sieves arranged in the order of decreasing opening sizes. The size fractions of aggregate samples retained on each sieve are then converted into percent passing by weight of total aggregate and reported as aggregate gradation. The gradation from 2D digital images are, however, calculated based on percent passing by the number of particles rather than weight of aggregate that are smaller than a specific sieve size. Furthermore, the determination of gradation from digital images requires identification of the following parameters: a) appropriate sieve size definition (square or diagonal), b) optimal particle shape parameter to compare with sieve sizes, so that a gradation obtained from digital images will be as close as possible to the actual gradation obtained from the mechanical sieving (Ozen and Guler, 2014).

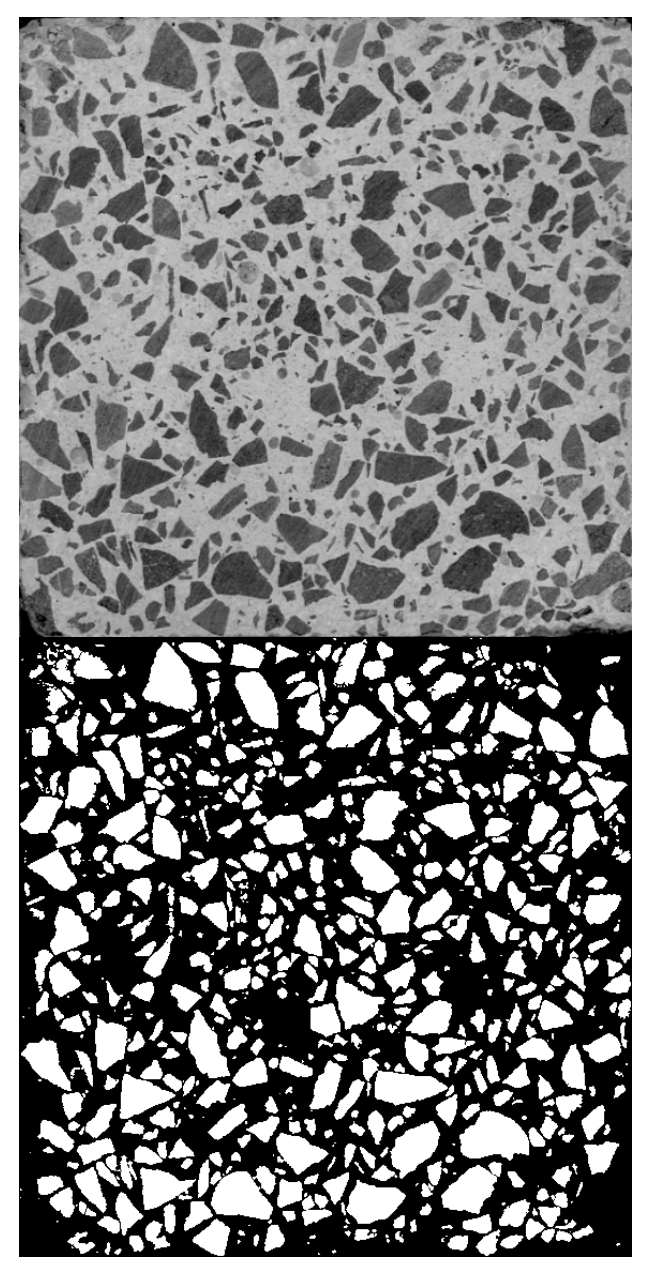

Fig. 2. Gray scale cross sectional image and binary image after erosion-dilation cycles

In the literature, there are a number of shape parameters calculated from 2D digital images to evaluate several aspects of aggregates particles. The authors have previously shown that maximum Feret diameter is the most suitable shape parameter producing a particle size distribution close to that of mechanical sieving when computed based on diagonal sieve opening for the concrete mixtures generated by the natural and crushed aggregates. These two aggregate sources are generally used for concrete production in the market and cover all possible variations in aggregate shapes for analysis. Natural aggregates are mostly in rounded in shape, whereas crushed aggregates become more angular after production (Ozen and Guler, 2014). As illustrated in Fig. 3, the maximum Feret diameter represents the length of the major axis of an aggregate particle that connects two points on its boundary with the farthest distance between them. Image Processing Toolbox of the MATLAB $\AA$ environment was used to label individual aggregate particles and calculate the maximum Feret diameter of each 
particle. Since crushed aggregates were used in this study, the sieve size of each aggregate particle was determined using the maximum Feret diameter in conjunction with the diagonal sieve openings, as seen in Fig. 4. The authors previously showed that the proposed methodology can accurately estimate the sample gradation with only minor errors for small size aggregates. Based on this finding, it can be presumed that the gradation computed from several 2D cross sections of a sample can well approximate its 3D or actual gradation.

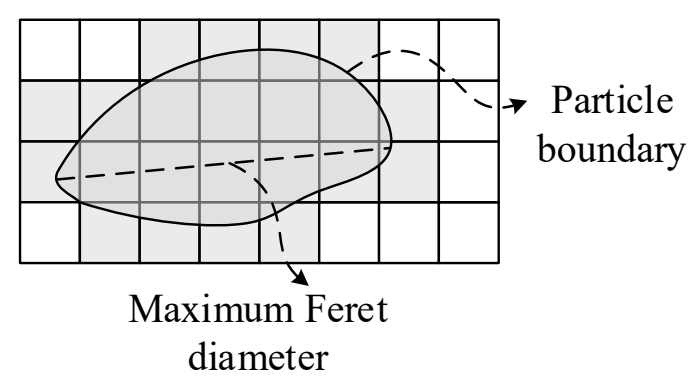

Fig. 3. Maximum Feret diameter of particle

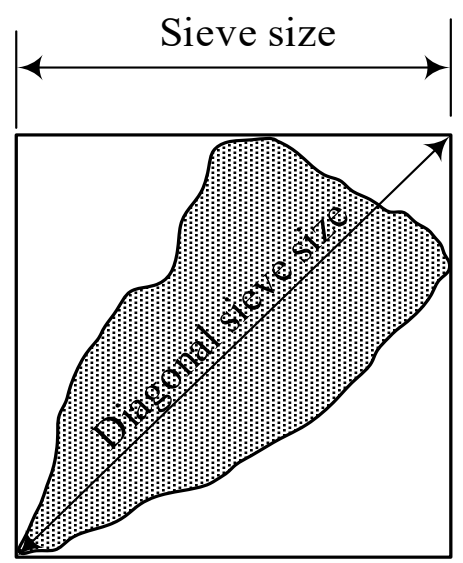

Fig. 4. Plan view of sieve opening with a particle cross section

\section{SEGREGATION ANALYSIS}

As described in the above section, segregation refers to the separation of coarse aggregates from the mortar in fresh concrete, yielding an inhomogeneous mixture (Mindess et al., 2002). Accordingly, coarse aggregate particles are expected to be uniformly distributed within the non-segregated mixtures. Based on the previous studies, it was decided that the distribution of aggregate particles retaining on $4.76 \mathrm{~mm}$ sieve will be sufficient to evaluate the degree of segregation in concrete mixtures (Navarrete and Lopez, 2016; Khayat et al.,
2007; Solak et al.; 2018, Barbosa et al., 2011). The proposed methodology herein is implemented in three steps as illustrated in Fig. 5. The developed algorithm in MATLAB $^{\circledR}$ environment is able to complete all the computational steps in less than minute for the analysis of a sample. In the first step, the optimum threshold is calculated to perform the gray scale thresholding. Then, the number of erosion-dilation cycles is applied to remove speckles and separate overlapping particles in the thresholded images. In the second step, aggregate particles are labelled, the maximum Feret diameter of each aggregate particle is calculated and compared with the diagonal sieve openings to determine the percent passing of aggregate particles. Next, fractional digital images are generated displaying only the aggregate particles of a specific sieve size equal to and larger than 4.76 $\mathrm{mm}$. In the final step, segregation index of the specimen $(S I)$ is calculated using Eq. (1-5).

$$
F S I_{i j}=\max \left(R I_{i j}, C I_{i j}\right)
$$

where $F S I=$ fractional segregation index calculated from the maximum of row segregation index $(R I)$ and column segregation index $(C I) . i=$ cross section number from 1 to $3 ; j=$ fraction number from 1 to $n ; n=$ number of sieve sizes equal to and larger than $4.76 \mathrm{~mm}$. For instance, $\mathrm{n}=2$ for mix design 1 because only $4.76 \mathrm{~mm}$ and $9.5 \mathrm{~mm}$ sizes are used in the analysis.

To calculate $R I$ and $C I$ from fractional images, first the number of particle pixels in each fractional image are calculated. If all the aggregate particles are spatially distributed at equal distances within the cross section, it should be expected that a single size fraction must also be equally spaced; as a result of this, the pixels belonging to aggregates are located at spatially equal distances in the rows and columns of the fractional image. The methodology used to detect the spatial distances is shown in Fig. 6. The straight line $(O A)$ represents the pixel distributions expected for a homogeneous mixture in which aggregates are distributed at nearly equal distances in a fractional image, and can be computed by cumulatively summing the number of pixels in each row and column by taking the upper left corner as the origin of the image. When a certain level of inhomogeneity exists in the cross section, the calculated sum of the pixels will start to deviate from the straight line and form a curve such as $(O M A)$ in Fig. 6. For an actual concrete cross section, the calculated number of pixels in the rowwise and column-wise directions will be similar to ones as shown in Fig 7 and 8. The amount of deviation from the straight line depends on the level of inhomogeneity of the cross section, and the calculated curve can cross the straight line depending on the characteristic of the spatial distribution of aggregate particles. 


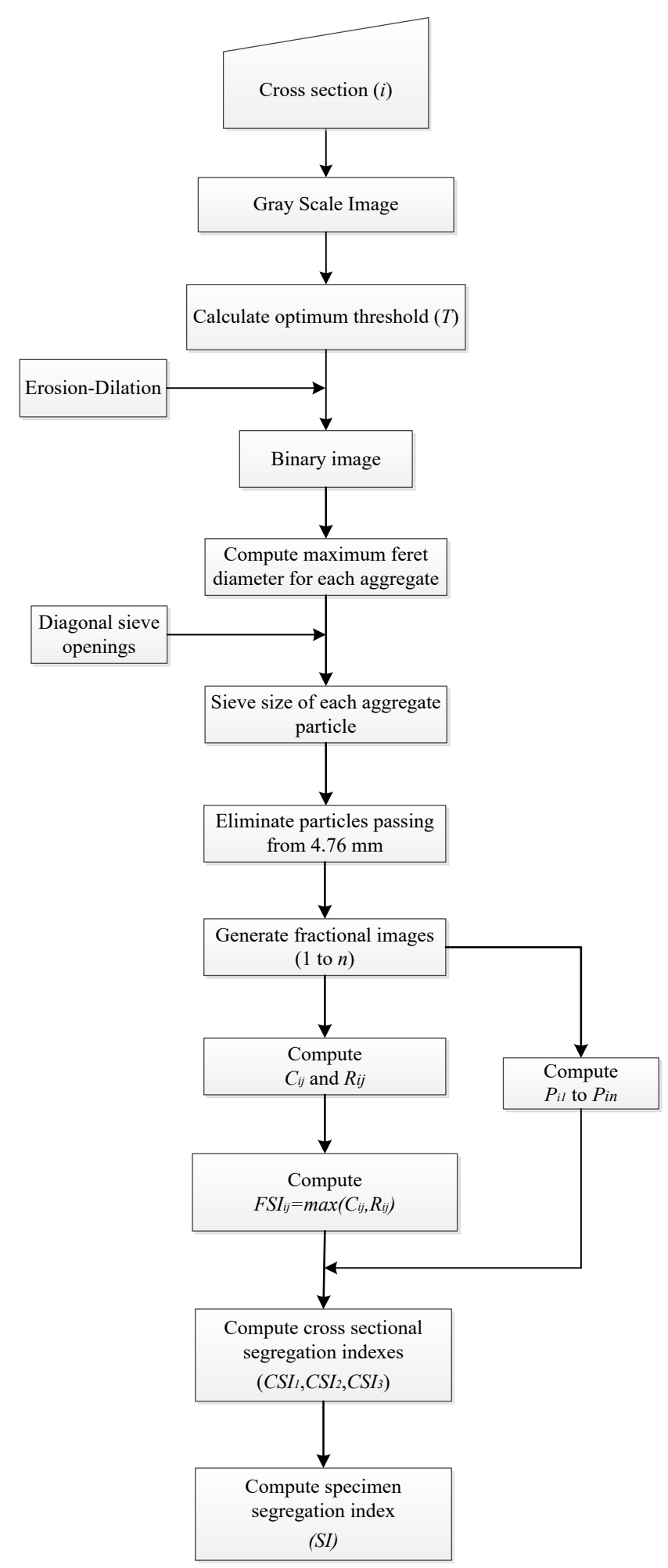

Fig. 5. Proposed algorithm to determine segregation of concrete samples

To calculate the segregation indices in the row-wise and column-wise directions, the percent ratio is simply calculated between the gray region underneath the straight line $(O A)$ and the shaded (triangle) region, which represents the maximum deviation for a segregated mixture, using the following relations;

$$
\begin{aligned}
& R I_{i j}=100 A_{r} /\left(N_{r} N_{f r} / 2\right) \\
& C I_{i j}=100 A_{c} /\left(N_{c} N_{f r} / 2\right)
\end{aligned}
$$

where $A_{r}$ and $A_{c}=$ area between actual and theoretical curves (see Fig. 7 and 8); $N_{f r}=$ total number of pixels within aggregate particles of fractional image; and $N_{r}$, $N_{c}=$ number of rows and columns in the fractional image, respectively.

In Eq. (2) and (3), the denominators represent hypothetically the maximum areas that can occur between actual and theoretical curves when maximum segregation level is reached in the mixture. Theoretically, RI and CI values range between 0 and 100. Ideally, if the particles are equally spaced by their spatial coordinates, the curves for the theoretical and actual pixel distributions will coincide, leading $R I$ and $C I$ values equal to zero as $A_{r}$ and $A_{c}$ are approaching to zero. On the other hand, as the distribution of aggregate particles deviate from the idealized case, the actual pixel distribution graphs will start shifting from the straight (theoretical) line; as a result, $R I$ and $C I$ values will increase. After determining the fractional segregation indices, the following equations are used to calculate the cross sectional segregation indices $(C S I S)$ and the overall segregation index of the specimen $(S I)$.

$$
\begin{aligned}
& C S I_{i}=\sum_{j=1}^{n} P_{i j} F S I_{i j} \\
& S I=\max \left(C S I_{1}, C S I_{2}, C S I_{3}\right)
\end{aligned}
$$

where $P_{i l}$ to $P_{i n}$ are the percent normalized retaining for each size fraction calculated from the digital image analysis and account for the relative proportions of sieve sizes in the mixture and calculated based on percent pixels of particles retaining on each sieve size (i.e. $P_{i 1}+P_{i 2}+$ $\ldots+P_{\text {in }}=100 \%$ ). For instance, for the first cross sectional image of Fig. 9, total number of particle pixels retaining on $4.76 \mathrm{~mm}$ and $9.5 \mathrm{~mm}$ sieves is 192,502 (see Fig. 10); therefore, $P_{11}=23 \%$ and $P_{12}=77 \%$ (see Table 2). 


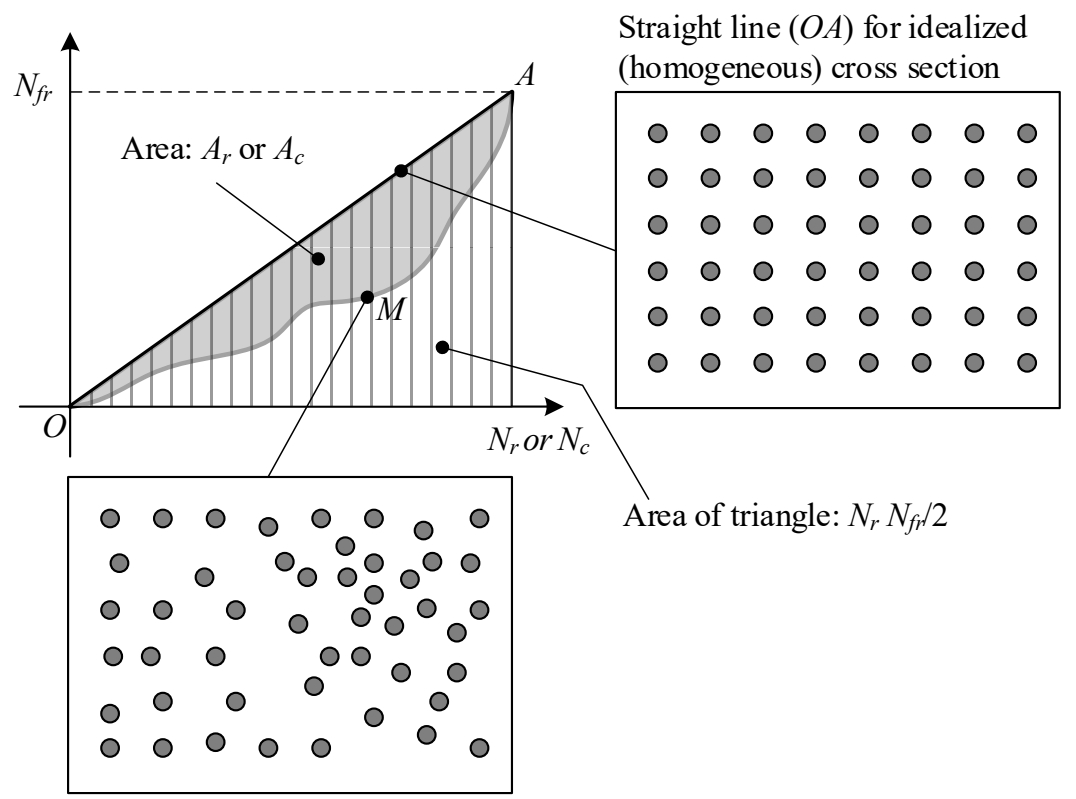

Curve $(O M A)$ for segregated cross section

Fig. 6. Method to calculate segregation index from 2D cross section image

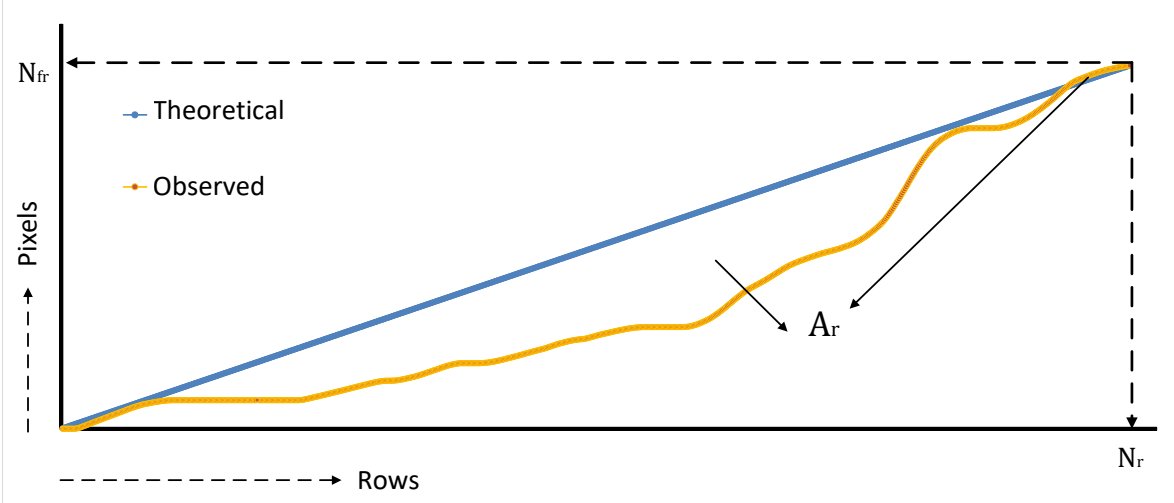

Fig. 7. Theoretical and actual distributions of particle pixels in fractional image along row-wise direction

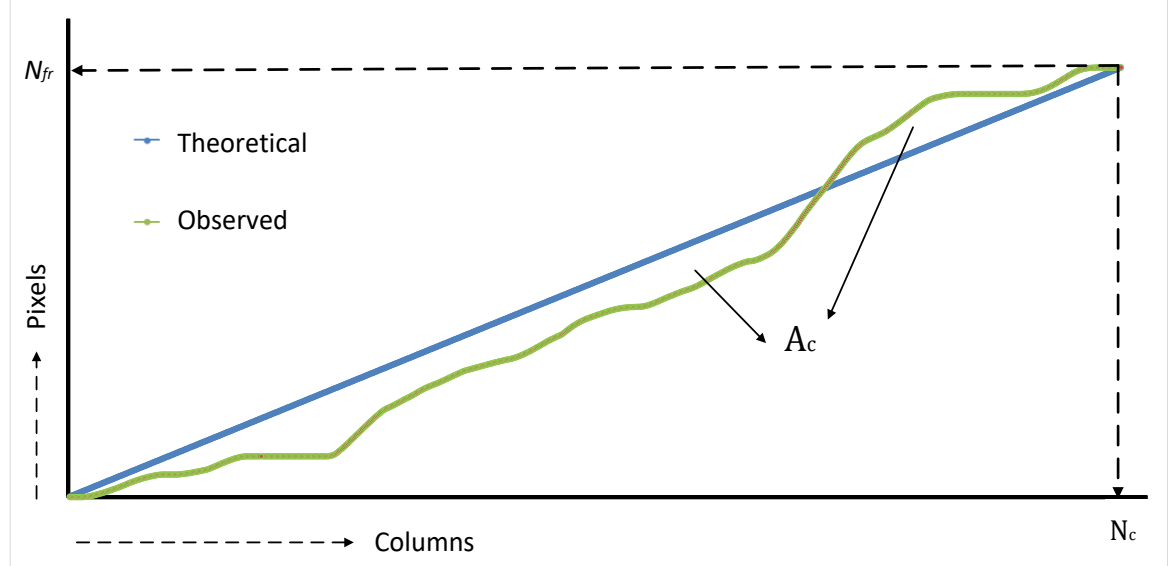

Fig. 8. Theoretical and actual distributions of particle pixels in fractional image along column-wise direction 


\section{RESULTS AND DISCUSSION}

To carry out segregation analyses, first the optimum threshold algorithm was implemented to convert gray scale cross sectional images into binary images. The optimum threshold was calculated separately for the three cross sections of the test samples. The next step was to calculate the maximum Feret diameters of the aggregates particles from the binary images produced after thresholding. The maximum Feret diameters were then compared with the diagonal sieve openings, so that the size distribution of all size fractions can be determined. In order to perform such analysis, the maximum Feret diameters calculated in pixels were converted into actual dimensions, i.e., unit of millimeters, using the known resolutions of the images and then compared with the diagonal sieve openings. Fractional images were then generated displaying only aggregates retaining on each sieve size equal to and larger than $4.76 \mathrm{~mm}$.

Fig. 9 shows binary cross sectional images of a specimen produced for mix design 1. For brevity, the fractional images and pixel distribution curves will be presented for one of the cross sectional images. Fig. 10 illustrates the fractional images of the first cross section in Fig. 9 together with the theoretical and actual distribution of particle pixels through the rows and columns of images. As it can be seen, the actual pixel distributions of particles retaining on $4.76 \mathrm{~mm}$ sieve almost coincide with the theoretical distributions. However, for $9.5 \mathrm{~mm}$ sieve fraction, the actual distribution deviated from the straight line because of the fact that the particles are not equally distributed in the fractional image as can be seen from Fig. 10, instead they seem to concentrate on the upper and lower parts of the cross section.
Cross Section I

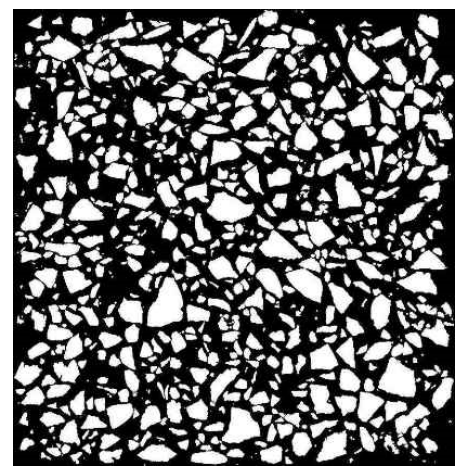

Cross Section II

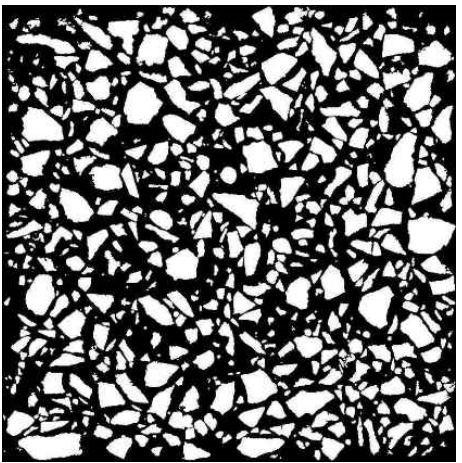

Cross Section III

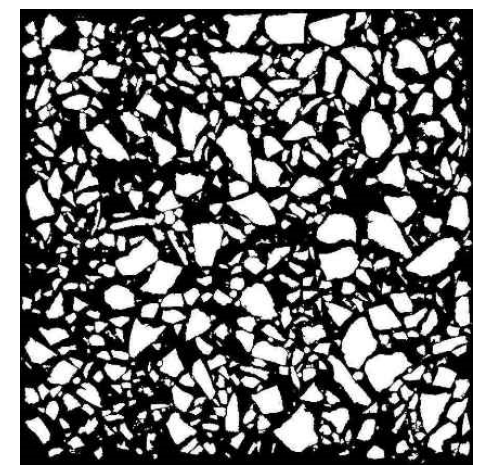

Fig. 9. Thresholded images for specimen of mix design 1
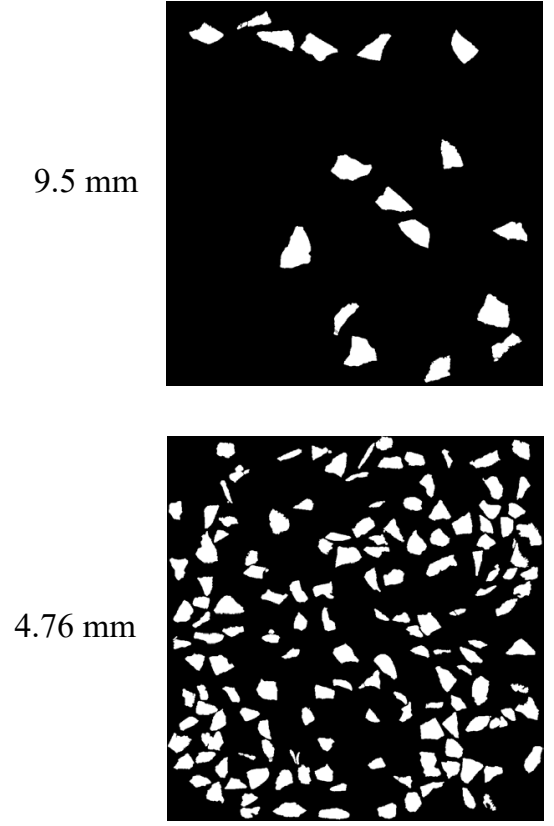
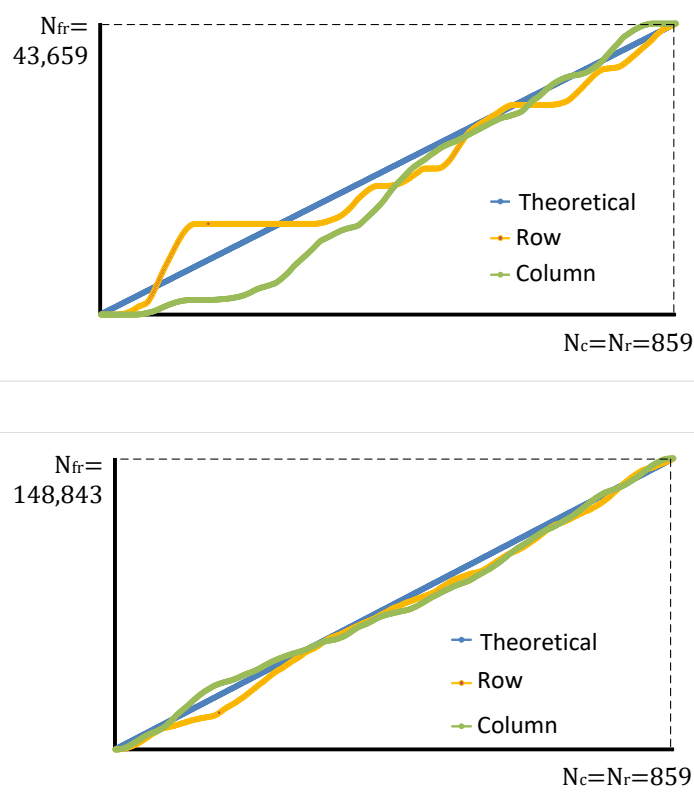

Fig.10. Fractional images of the first cut section for specimen of mix design 1 
In Table 2, the calculated values of RI, CI, FSI, CSI and $S I$ are listed for the three cross sectional images of this specimen shown in Fig. 9. In general, particles retaining on $4.76 \mathrm{~mm}$ sieve results in the lowest FSI values. On the other hand, the highest FSI values were obtained for $9.5 \mathrm{~mm}$ sieve size. After multiplying by the normalized percent retaining values $\left(P_{I i}\right)$, while the lowest cross sectional segregation index $(C S I)$ was obtained for the first cut surface, the highest $C S I$ was obtained for the third cut surface. As a result, the segregation index $(S I)$ of this specimen was found to be $11.4 \%$, which is the maximum of the computed $C S I$ values.

Table 2. FSI, CSI and SI values for specimen of mix design 1

\begin{tabular}{|c|c|c|c|c|c|c|c|c|c|c|c|c|}
\hline \multirow{2}{*}{$\begin{array}{l}\text { Sieve } \\
(\mathrm{mm})\end{array}$} & \multicolumn{4}{|c|}{ Cross Section I } & \multicolumn{4}{|c|}{ Cross Section II } & \multicolumn{4}{|c|}{ Cross Section III } \\
\hline & $R I$ & $C I$ & $F S I$ & $\begin{array}{l}P_{l j} \\
(\%)\end{array}$ & $R I$ & $C I$ & $F S I$ & $\begin{array}{l}P_{2 j} \\
(\%)\end{array}$ & $R I$ & $C I$ & $F S I$ & $\begin{array}{l}P_{3 j} \\
(\%)\end{array}$ \\
\hline 9.5 & 9.9 & 14.8 & 14.8 & 23 & 12.4 & 9.3 & 12.4 & 29 & 8.4 & 19.1 & 19.1 & 43 \\
\hline 4.76 & 4.7 & 4.5 & 4.7 & 77 & 5.8 & 4.3 & 5.8 & 71 & 5.6 & 4.2 & 5.6 & 57 \\
\hline CSI $(\%)$ & \multicolumn{4}{|c|}{7.0} & \multicolumn{4}{|c|}{7.7} & \multicolumn{4}{|c|}{11.4} \\
\hline$S I(\%)$ & \multicolumn{12}{|c|}{11.4} \\
\hline
\end{tabular}

Fig. 11 shows binary cross sectional images of a specimen prepared for mix design 2. Fig. 12 illustrates the fractional images as well as theoretical and actual distributions of particle pixels for the second cross sectional image in Fig. 11. The calculated segregation indexes FSI, CSI and SI for these cross sections can be found in Table 3. A slight deviation can be observed for $4.76 \mathrm{~mm}$ in row-wise direction and for $12.7 \mathrm{~mm}$ in column-wise direction. From the fractional images shown in Fig. 12, it can be seen that the homogeneity level in row-wise direction is slightly better than in column-wise direction for $4.76 \mathrm{~mm}$; conversely it is slightly better in column-wise direction than in row-wise direction for $12.7 \mathrm{~mm}$. On the other hand, the distribution curves considerably deviated from the theoretical lines in row-wise direction for $9.5 \mathrm{~mm}, 12.7 \mathrm{~mm}$. and $19.1 \mathrm{~mm}$. For the first and the third cut sections in Fig 11, particles retaining on $4.76 \mathrm{~mm}$ sieve results in the lowest FSI values (see Table 3). For the second cut section, particles retaining on $12.7 \mathrm{~mm}$ has the lowest $F S I$ value while the highest values were obtained for $19.1 \mathrm{~mm}$ sieve size. It can be noticed that even though particles retaining on $19.1 \mathrm{~mm}$ resulted in the highest $F S I$ values for all the cut sections analyzed, these particles do not still dominate the overall segregation index as they constitute the smallest proportion in the mixture; thereby resulting in the lowest $P_{i j}$ values. For the same test sample, the largest $C S I$ value was obtained from the first cross section, hence the sample segregation index $(S I)$ is reported as $16.3 \%$.
Cross Section I

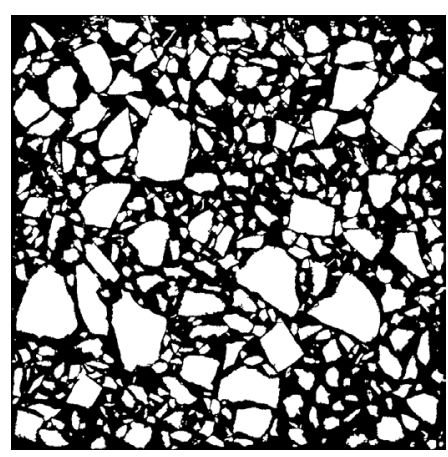

Cross Section II

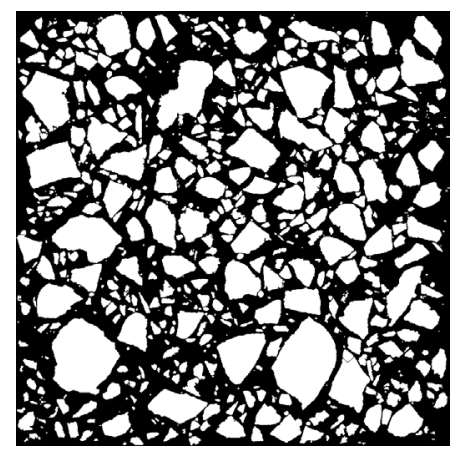

Cross Section III

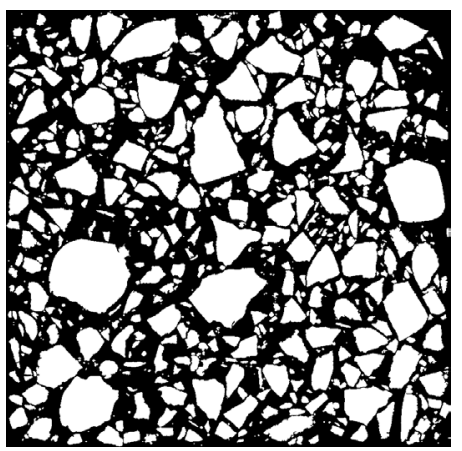

Fig. 11. Thresholded images for specimen of mix design 2 

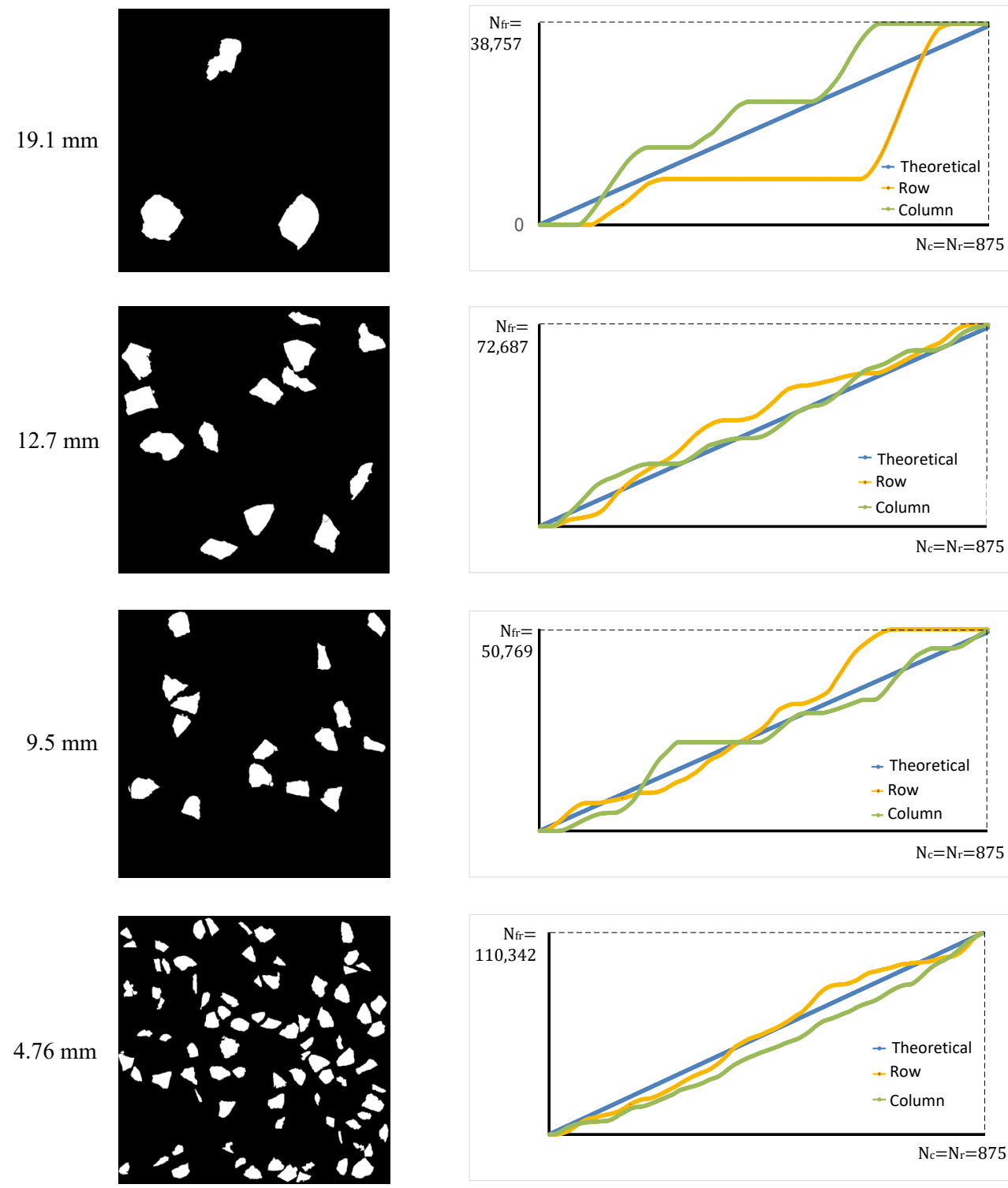

Fig. 12. Fractional images of the second cut section for specimen of mix design 2

Table 3. FSI, CSI and SI values for specimen of mix design 2

\begin{tabular}{|c|c|c|c|c|c|c|c|c|c|c|c|c|}
\hline \multirow{2}{*}{$\begin{array}{l}\text { Sieve } \\
(\mathrm{mm})\end{array}$} & \multicolumn{4}{|c|}{ Cross Section I } & \multicolumn{4}{|c|}{ Cross Section II } & \multicolumn{4}{|c|}{ Cross Section III } \\
\hline & $R I$ & $C I$ & $F S I$ & $\begin{array}{l}P_{1 j} \\
(\%)\end{array}$ & $R I$ & $C I$ & $F S I$ & $\begin{array}{l}P_{2 j} \\
(\%)\end{array}$ & $R I$ & $C I$ & $F S I$ & $\begin{array}{l}P_{3 j} \\
(\%)\end{array}$ \\
\hline 19.1 & 20.7 & 34.0 & 34.0 & 13 & 36.1 & 20.1 & 36.1 & 14 & 30.4 & 32.3 & 32.3 & 14 \\
\hline 12.7 & 14.1 & 12.4 & 14.1 & 30 & 12.0 & 6.4 & 12.0 & 26 & 9.8 & 26.8 & 26.8 & 21 \\
\hline 9.5 & 8.9 & 26.4 & 26.4 & 17 & 14.4 & 8.7 & 14.4 & 19 & 10.8 & 9.0 & 10.8 & 23 \\
\hline 4.76 & 7.2 & 7.8 & 7.8 & 40 & 6.7 & 12.8 & 12.8 & 41 & 8.1 & 4.5 & 8.1 & 42 \\
\hline CSI (\%) & \multicolumn{4}{|c|}{16.3} & \multicolumn{4}{|c|}{16.2} & \multicolumn{4}{|c|}{16.0} \\
\hline$S I(\%)$ & \multicolumn{12}{|c|}{16.3} \\
\hline
\end{tabular}


To verify the sensitivity of the proposed method, artificial images were generated by algorithmically redistributing aggregate particles in the cross section images of mix design 1 and 2. During this process, overlapping or connected particles were avoided. As many as 5 artificial sections were generated and plotted together with their original cross sections as shown in Fig. 13 and 14 by changing the spatial location of aggregate fractions used in the calculation of segregation index. For each mix design, the first sections (I1) were so produced that the section's homogeneity level is better than their original ones, and the other sections were simply produced by progressively dislocating each size fraction to increase the segregation level.

Generated artificial images for cross section III of mix design 1 are shown in Fig. 13, and results of the corresponding CSI values are given in Table 4 . The calculated percent fractions $\left(P_{3 j}\right)$ for $4.76 \mathrm{~mm}$ and $9.5 \mathrm{~mm}$ sieves were found to be $57 \%$ and $43 \%$, respectively (see
Table 2). It can be observed that section I2 produces a slightly higher CSI index of $9.9 \%$ due to agglomeration of $9.5 \mathrm{~mm}$ particles at nearly center of the section. The section I3, on the other hand, shows even higher segregation with a CSI of $20.4 \%$ by the distribution of the coarse fraction on the boundary of the cross section. When the segregation indexes of I 2 and I 3 are carefully compared, it can be noticed that particles retaining on $4.76 \mathrm{~mm}$ sieve generates better homogeneity in $\mathrm{I} 2$ as compared to both $4.76 \mathrm{~mm}$ and $9.5 \mathrm{~mm}$ sizes in $\mathrm{I} 3$, hence resulting in a significantly higher $C S I$ value than $\mathrm{I} 2$. When only the $9.5 \mathrm{~mm}$ fraction is separated from the rest of the particles, CSI value was found to be $27.0 \%$. For the last image, however, the largest CSI value of $28.6 \%$ was obtained when $4.76 \mathrm{~mm}$ is separated from the other fractions, and the largest and the smallest fractions are accumulated at the bottom of the section. The analysis of the generated artificial images indicate that the level of segregations identified by visual inspection agrees well with the calculated cross sectional index values.
Original

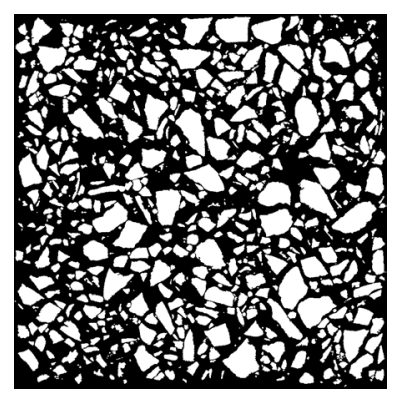

I3

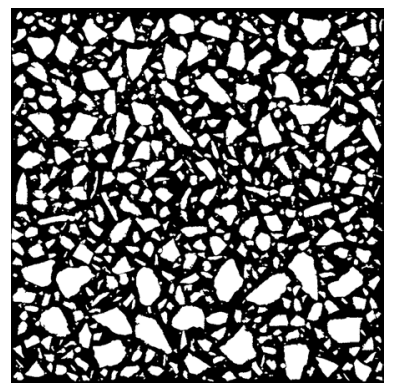

I1

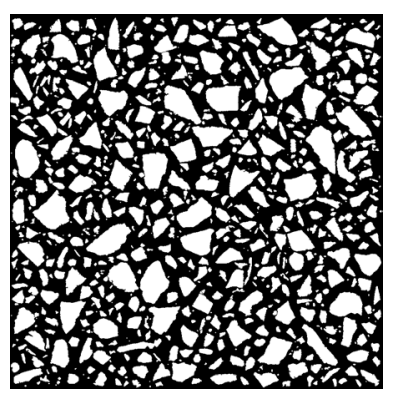

I4

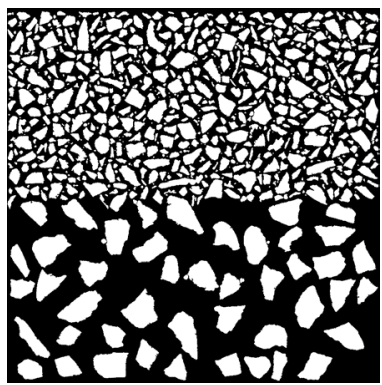

$\mathrm{I} 2$

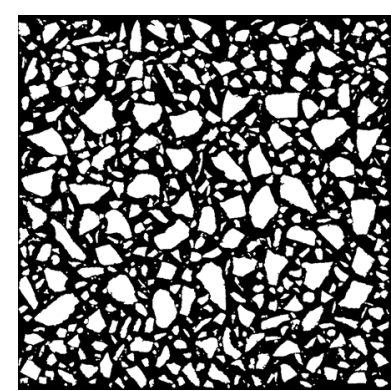

I5

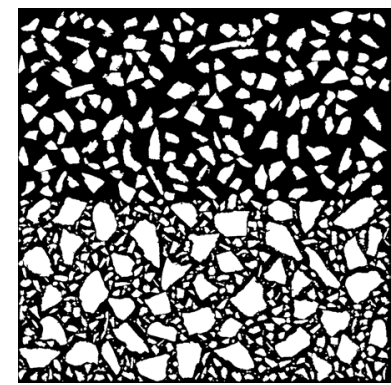

Fig. 13. Imaginary images generated for cross section 3 of mix design 1

Table 4. Segregation index for the generated images in Fig. 13

\begin{tabular}{|c|c|c|c|c|c|c|c|}
\hline \multirow{2}{*}{$\begin{array}{l}\text { Sieve } \\
(\mathrm{mm})\end{array}$} & \multirow{2}{*}{$\begin{array}{l}P_{3 j} \\
(\%)\end{array}$} & \multicolumn{6}{|c|}{ FSI (\%) } \\
\hline & & Original & I1 & I2 & I3 & I4 & I5 \\
\hline 9.5 & 43 & 19.1 & 10.0 & 17.4 & 22.7 & 44.9 & 45.9 \\
\hline 4.76 & 57 & 5.6 & 4.7 & 4.3 & 18.6 & 13.5 & 15.5 \\
\hline \multicolumn{2}{|c|}{ CSI (\%) } & 11.4 & 7.0 & 9.9 & 20.4 & 27.0 & 28.6 \\
\hline
\end{tabular}


A similar strategy was also used to generate the artificial images for cross section II of mix design 2 as shown in Fig. 14. PI values of the size fractions were ranged from $14 \%$ to $41 \%$, as can be seen in Table 5 . A careful inspection of the original cross section reveals the grouping of coarse size fraction nearly at the left side of the section, resulting in a higher segregation level. It can be observed that section I1 with a CSI of $15.8 \%$ has slightly more homogenous aggregate distribution as compared to the original section with a CSI of $16.2 \%$. Section 12 resulted in a higher segregation level than section I1 with a CSI of $17.9 \%$. When the largest size fraction $(19.1 \mathrm{~mm})$ is agglomerated within the center of the cross section in $\mathrm{I} 3$, the calculated CSI value becomes significantly higher than the CSI of the original cross section. Segregation index of the cross section was further increased in I4 by locating some largest particles at the top, one size smaller to the bottom and the intermediate sizes in the middle of the cross section. For this section, the calculated segregation level was found to be $29.7 \%$. The highest segregation of $35.3 \%$ was achieved by locating the larger fractions to the bottom of the cross section in section I5.
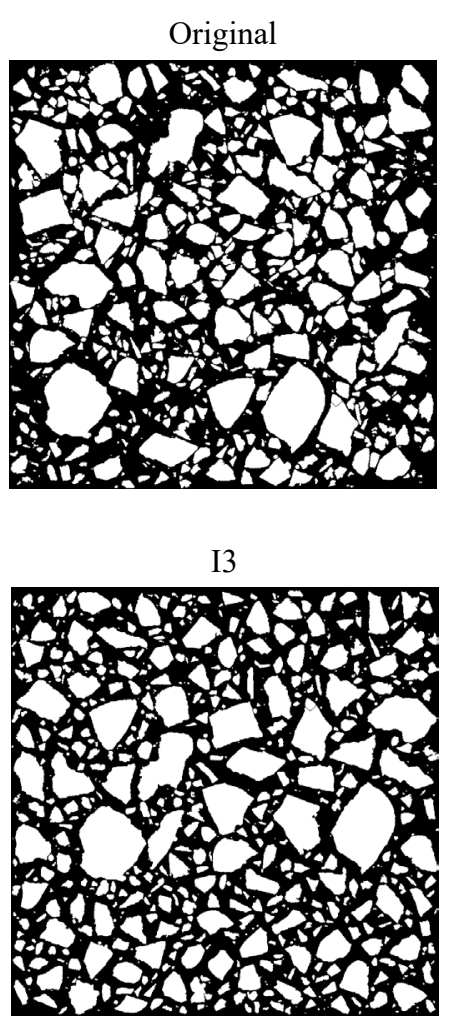
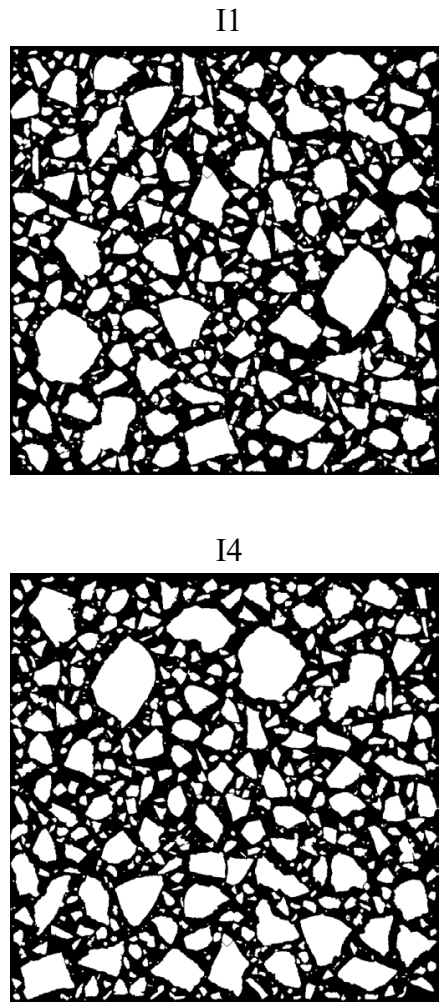

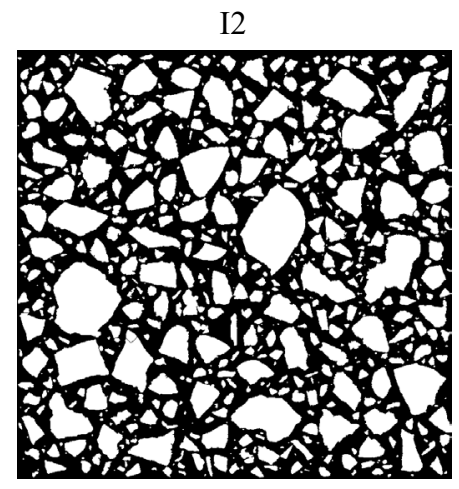

$\mathrm{I} 5$

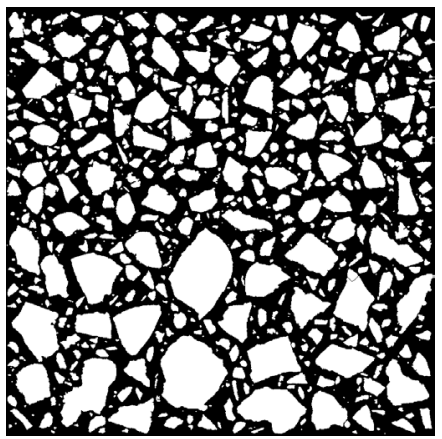

Fig. 14. Imaginary images generated for cross section 2 of mix design 2

Table 5. Segregation index for generated images shown in Fig. 14

\begin{tabular}{cccccccc}
\hline \multirow{2}{*}{$\begin{array}{c}\text { Sieve } \\
(\mathrm{mm})\end{array}$} & $P_{2 j}$ & \multicolumn{6}{c}{ FSI (\%) } \\
\cline { 3 - 8 } & Original & I1 & I2 & I3 & I4 & I5 \\
\hline 19.1 & 14 & 36.1 & 39.7 & 35.6 & 37.6 & 57.9 & 55.2 \\
\hline 12.7 & 26 & 12.0 & 12.3 & 11.1 & 35.0 & 35.8 & 46.2 \\
\hline 9.5 & 19 & 14.4 & 25.4 & 31.1 & 16.6 & 35.0 & 17.0 \\
\hline 4.76 & 41 & 12.8 & 5.3 & 10.0 & 19.4 & 13.7 & 30.0 \\
\hline \multicolumn{2}{c}{$C$ CSI (\%) } & 16.2 & 15.8 & 17.9 & 25.5 & 29.7 & 35.3 \\
\hline
\end{tabular}


It should be noticed that the computed CSI value for I5 of Fig. $13(28.6 \%)$ is lower than for I5 of Fig. 14 $(35.3 \%)$. Even though these two sections are not directly comparable, due to having different aggregate fractions, the larger CSI value for I5 of Fig. 14 can be justified by carefully inspecting the segregation levels introduced by the $12.7 \mathrm{~mm}$ and $19.1 \mathrm{~mm}$ aggregate fractions. While these two factions do not exist in the mixture of Fig. 13., a FSI of $55.2 \%$ from $19.1 \mathrm{~mm}$ and $46.2 \%$ from $12.7 \mathrm{~mm}$ are added to the segregation level of the mixture by their relative percentages in Fig. 14. Besides, there is also an appreciable amount of fractional segregation that can be observed from the $4.76 \mathrm{~mm}$ fraction in Fig. 14 with a FSI of $30.0 \%$.

The analysis of the example mix designs shows that a qualitative rating of mixture segregation level is possible. The calculated segregation levels for the artificial images of mix design 1 range from $7.0 \%$ to $28.6 \%$, while $11.4 \%$ was found for the original design cross section (see Table 4). The aggregate distributions in these sections indicate that sections I1 and I2 can be among the possibly generated segregation levels in the field with $C S I$ values of $7.0 \%$ and $9.9 \%$, respectively. On the other hand, sections I3 through I5 seem unlikely to be observed in the field, unless special effort is given to obtain such a high segregation level in the mixture. In Table 2, the CSI values for the three cut sections of mix design 1 ranged between $7.0 \%$ and $11.4 \%$ with a relatively homogenous distribution for the first cut section. It should be noticed that the first cut section $(C S I=7.0 \%)$ has distribution characteristics nearly equal to the artificially generated cross section I1 based on the comparison of their CSI values. However, a close inspection of the third section reveals that the mix design 1 was, in fact, produced with a moderately segregated mixture, resulting in an overall segregation level of $(S I)$ of $11.4 \%$.

For the mix design 2, the artificial images were generated for the second cut section, for which the calculated CSI values ranged between $16.0 \%$ and $16.3 \%$ as given in Table 3 . The lowest CSI value for the artificially generated mixture was found to be $15.8 \%$ for section I1, as seen in Table 5. However, the $S I$ value for the second cut section of this mixture is slightly higher (16.2\%) than for I1 and very close to I2 indicating that the second cut section distribution characteristics somewhere between I1 and I2. However, the segregation levels for the remaining sections I3, I4 and I5 seem significantly more heterogeneous than the original cross (cut) section, hence unlikely to appear in the field.

The benefit of the proposed method relies on its simplicity to quantitatively determine the mixture segre- gation level based on 2D cut sections that are easily obtained using a document scanner. The method can also be applied for cylindrical core samples of concrete taken in the field. In this case, because only one vertical cut section can be taken for image analysis due to size limitations, the results for both gradation and segregation analyses can be statistically improved by increasing the number of core samples; at least three samples to be equivalent to one cubic sample used in this study. Using the described procedure, the determination of mixture segregation index can be easily performed for a hardened concrete sample to evaluate its segregation without any personal judgement or subjective rating based on visual inspection methods. In this way, the calculated $S I$ index can be used as a quality control criterion for hardened concrete after the placement process. As indicated earlier, excessive segregation in the mixture can dramatically reduce the strength of hardened concrete by poor load transfer between mortar and the aggregate skeleton at micro-structure level. Since segregation is characterized at micro-structural level in the proposed method, i.e., spatial distribution of size fractions, further investigations can be conducted to establish a possible correlation between segregation level and strength properties of hardened concrete.

\section{CONCLUSION}

The main objective of this paper is to evaluate segregation of Portland cement concrete samples using twodimensional cross sectional images. Unlike the previous works on mixture segregation, this study considered the spatial distribution of coarse aggregate fractions within the concrete cross sections. However, by only looking at the distribution of coarse aggregate may not always help obtain the actual segregation level of mixture. Even if the coarse aggregates are equally distributed in the cement paste, the small size fractions can still form clusters and result in segregation at relatively small scale. Based on the analyses of segregation levels for the test mixtures, the following conclusions can be drawn:

- Degree of segregation in hardened Portland cement concrete can be successfully identified from two-dimensional digital images of cut sections.

- Segregation level depends highly on the percentage of large size aggregates in the mixture. In general, the potential of generating segregated mixtures is more likely for coarser mixtures than for fine graded mixtures. 
- Degradation in mixture's homogeneity is controlled by the combined effect of particle distribution and their relative proportions in the mixture. Hence, any measure of segregation used must account for not only spatial distribution of aggregate particles, but also their size fractions in the mixture.

- $\quad$ Proposed segregation index is determined without any use of personal judgment or subjective rating procedure; therefore, it can be used as a quality control parameter for hardened concrete samples to evaluate the degree of homogeneity achieved after production.

- The outcome of the study can be extended further to investigate a possible correlation between the developed index and the strength properties of concrete.

\section{REFERENCES}

Barbosa FS, Beaucour AL, Farage MC, Ortola S (2011). Image processing applied to the analysis of segregation in lightweight aggregate concretes. Constr Build Mater 25(8):3375-81.

Erdem S (2014). X-ray computed tomography and fractal analysis for the evaluation of segregation resistance, strength response and accelerated corrosion behavior of self-compacting lightweight concrete. Constr Build Mater 61:10-7.

Fang C, Labi S (2007). Image-processing technology to evaluate static segregation resistance of hardened selfconsolidating concrete. Transp Res Record 2020:1-9.

Fernlund JM, Zimmerman RW, Kragic D (2007). Influence of volume/mass on grain-size curves and conversion of image-analysis size to sieve size. Eng Geol 90(3-4):124-37.

Ferraris CF, Koehler E, Amziane S (2008). Report on measurements of workability and rheology of fresh concrete. ACI Committee 238, Report 238.1R-08.

He, H, Courard, L, Pirard, E, Michel, F (2016). Shape analysis of fine aggregates used for concrete. Image Anal Stereol 35(3): 159-66.
Johnson D, Johnson G, Robertson IN (2010). Quantifying segregation in self-consolidating concrete through image analysis. Department of Civil and Environmental Engineering, University of Hawaii, Research Report $\mathrm{UHM} / \mathrm{CEE} / 10-14$.

Khayat KH, Vanhove Y, Pavate TV, Jolicoeur C (2007). Multi-electrode conductivity method to evaluate static stability of flowable and self-consolidating concrete. ACI Mater J 104(4):424-33.

Lee JR, Smith ML, Smith LN (2007). A new approach to the three-dimensional quantification of angularity using image analysis of the size and form of coarse aggregates. Eng Geol 91(2-4):254-64.

Mesbah HA, Yahia A, Khayat KH (2011). Electrical conductivity method to assess static stability of self-consolidating concrete. Cement Concrete Res 41(5):45158.

Mindess S, Young JF, Darwin D (1981). Concrete. Prentice-Hall, NJ.

Mora CF, Kwan AK (2000). Sphericity, shape factor, and convexity measurement of coarse aggregate for concrete using digital image processing. Cement Concrete Res 30(3):351-58.

Navarrete I, Lopez M (2016). Estimating the segregation of concrete based on mixture design and vibratory energy. Constr Build Mater 122:384-90.

Nichols AB, Lange DA (2006). 3D surface image analysis for fracture modeling of cement-based materials. Cement Concrete Res 36(6):1098-107.

Ozen M, Guler M (2014). Assessment of optimum threshold and particle shape parameter for the image analysis of aggregate size distribution of concrete sections. Opt Laser Eng 53:122-32.

Peterson K, Swartz R, Sutter L, Van Dam T (2001). Hardened concrete air void analysis with a flatbed scanner. Transp Res Record 1775:36-43.

Solak AM, Tenza-Abril AJ, Baeza Brotons F (2018). Image analysis applications for the study of segregation in lightweight concretes. Int J Comp Meth and Exp Meas 6(4):835-46.

Yang XW, Man HG, Tian RL (2010). Deformation measurement of concrete by white light digital image analysis in frequency domain. Appl Mech Mater 29:49601 . 